\title{
The PLANET-B mission
}

\author{
T. Yamamoto and K. Tsuruda \\ Institute of Space and Astronautical Science, 3-1-1 Yoshinodai, Sagamihara, Kanagawa 229-8510, Japan
}

(Received August 13, 1997; Revised January 28, 1998; Accepted February 5, 1998)

\begin{abstract}
PLANET-B is the Japanese Mars orbiter program. The spacecraft will begin its journey to Mars in July 1998, and will be inserted into Mars orbit in October 1999. It will collect data for at least one Martian year (about two Earth years). The primary objective of the program is to study the Martian aeronomy, with emphasis on the interaction of the Martian upper atmosphere with the solar wind. The periapsis altitude and the apoapsis distance are $150 \mathrm{~km}$ and 15 Mars radii, respectively. The dry weight of the orbiter is $258 \mathrm{~kg}$ including 15 scientific instruments. Advanced technologies are employed in the design of the spacecraft in attempt to overcome the mass limitation. This paper describes the scientific objectives of the PLANET-B program and the outline of the observation scenario.
\end{abstract}

\section{Mission Objectives}

The PLANET-B spacecraft (Tsuruda et al., 1996; see Plate 1) will be placed into an ecliptic orbit around Mars with a periapsis altitude of $150 \mathrm{~km}$ and an apoapsis of 15 Mars radii in October 1999. The primary objective of the program is to study Martian aeronomy, putting emphasis on the interaction of the upper atmosphere with the solar wind. Table 1 summarizes the parameters of the spacecraft. Some details of the spacecraft system are described by Tsuruda et al. (1996).

The Martian upper atmosphere remains unexplored by spacecraft equipped with a set of comprehensive instruments. Except for the two Viking landers, the Phobos 2 periapsis altitude of $850 \mathrm{~km}$ is the lowest, but it is still too high for investigations of the most important altitude range of 150 $300 \mathrm{~km}$, where the Martian ionosphere has its peak electron density. The scarcity of in situ measurements of the Martian upper atmosphere has made it difficult to discuss its structure and the physical processes. In an attempt to cover the altitude range of interest, the periapsis altitude of the PLANET-B orbit is designed as low as $150 \mathrm{~km}$. The effects of the solar wind on the ionosphere would be largest at the sub-solar point where the dynamic pressure of the solar wind is maximum. The orbit is designed so that the periapsis comes close to the sub-solar point at least twice during the designed lifetime of the spacecraft.

The previous observations of magnetic field around Mars indicate the planet does not have an intrinsic magnetic field strong enough to stand off the solar wind far above its upper atmosphere. In this regard, Mars is similar to Venus. From the PVO (Pioneer Venus Orbiter) observations, the Venusian upper atmosphere is known to have a clear ionopause structure and its height is strongly controlled by the solar wind (Taylor, Jr. et al., 1979). The two in situ measurements of the Martian ionosphere profiles made by the two Viking landers, however, do not show a clear ionopause structure (Hanson et

Copy right $(\mathrm{C})$ The Society of Geomagnetism and Earth, Planetary and Space Sciences (SGEPSS); The Seismological Society of Japan; The Volcanological Society of Japan; The Geodetic Society of Japan; The Japanese Society for Planetary Sciences. al., 1977). This evidence suggests a different physical process operating at the Martian upper ionosphere. The mean distance to the sub-solar shock at Mars is larger than at Venus when normalized by the respective planetary radius (Slavin and Holzer, 1981). The clear dependence of the shock position on the solar cycle observed at Venus is not outstanding at Mars (Zhang et al., 1990, and Slavin et al., 1983). These results could be interpreted in terms of an indirect evidence for the presence of a weak but significant intrinsic magnetic field of Mars. The presence of the Martian intrinsic magnetic field is still an open question. The low periapsis altitude of the PLANET-B has a special advantage to give a final resolution to this problem.

The importance of the plasma measurements in the nightside tail region of Mars is demonstrated by the finding of escaping ionospheric ions with Phobos 2 (Lundin et al., 1989). The total mass flux and energy flux carried away by the escaping ions are so enormous that, if the escape occurs continuously, significant effects are expected on the evolution of the atmosphere. PLANET-B will carry out extensive and systematic observations with instruments to measure a wide energy range of ions in the tail region. The apoapsis of 15 Mars radii is suited for the observation in the tail. The combination of in situ measurements at the topside ionosphere and at the tail will give us key evidences for physical mechanisms relating to ionospheric ion escape at Mars.

Although the primary research area for the PLANET-B program is Martian aeronomy, the researches in other fields are also included. The visible camera is used as a tool for the Martian meteorology. It will be also used to take a photographs of the Martian surface with a moderate resolution of $100 \mathrm{~m}$ per pixel. The orbit of the spacecraft intersects several times with those of Phobos and Deimos. If we are lucky, close-up images of those satellites can also be obtained. The dust counter observes dust particles during the cruise phase and in the orbit of Mars. There is a possibility to detect a dust ring of Mars or set an upper limit of dust density around Mars.

In addition to the scientific objectives described above, the 


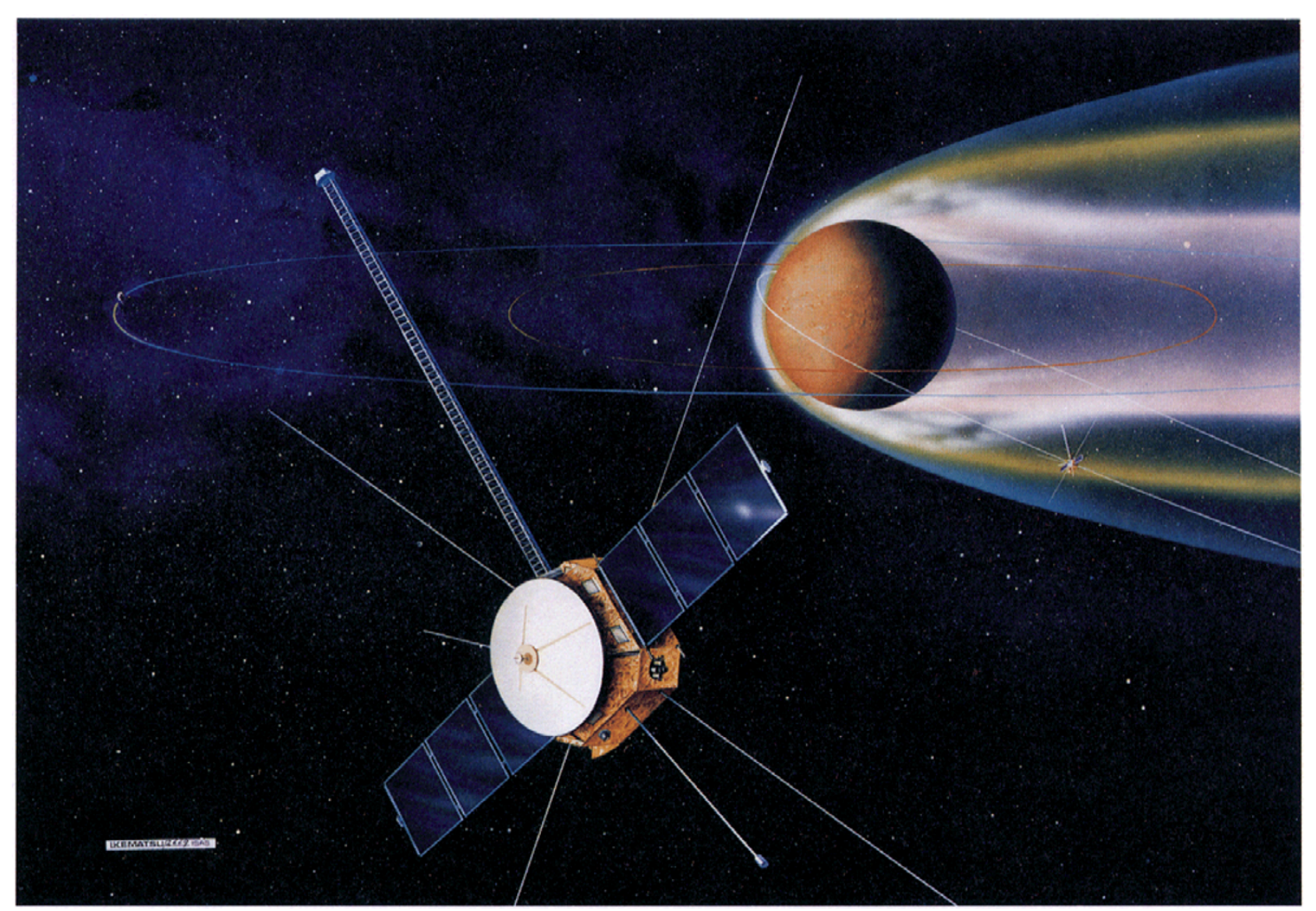

Plate 1. Artist's impression of PLANET-B and Mars.

Table 1. Outline of spacecraft.

\begin{tabular}{ll}
\hline Launch vehicle & M-V, solid propellant \\
Launch & July 1998 \\
Trans-Mars orbit insertion & December 1998, by bi-propellant \\
Mars orbit insertion & October 1999, by bi-propellant \\
Operation period & 2 Earth years or longer \\
Dry weight & $258 \mathrm{~kg}$ \\
Size & 1.8 meters in diameter \\
Attitude & Spin stabilized, Earth pointing, 8 sec spin period \\
Orbit in Mars & Close to ecliptic plane, 150 km periapsis, 15 Mars radii apoapsis \\
Telemetry & S/X band, 2 kbps minimum and 32 kbps maximum \\
Onboard data edition rate & 65 kbps to 64 bps \\
Onboard data recorder & 16 Mbytes \\
Science payload & About $33 \mathrm{~kg}, 15$ scientific instruments \\
Appendages & $5 \mathrm{~m}$ MAST, 2 pairs of $50 \mathrm{~m}$ tip-to-tip wire antennas $1 \mathrm{~m}$ boom \\
\hline
\end{tabular}

PLANET-B program has engineering objectives to develop key technologies for Japanese future planetary missions. The PLANET-B is scheduled to be launched with a M-V rocket. Its payload capability is moderate and one of the engineering challenges in the program is to realize a light-weight but high-performance spacecraft. The dry weight of the spacecraft is about $258 \mathrm{~kg}$, including 15 scientific instruments. Extremely light-weight onboard equipment both for the bus and scientific instruments are necessary to realize the science objectives within this mass limit. 
Table 2. List of PLANET-B instruments.

\begin{tabular}{|c|c|c|c|c|c|}
\hline \multicolumn{2}{|c|}{ Instrument } & \multirow[t]{2}{*}{ Measurement } & $\begin{array}{c}\text { Mass } \\
(\mathrm{kg})\end{array}$ & Remarks & \multirow[t]{2}{*}{$\begin{array}{l}\text { International } \\
\text { collaboration }\end{array}$} \\
\hline PSA & & & 5.9 & & \\
\hline ISA & Energetic ions & $10 \mathrm{eV}-20 \mathrm{keV} / \mathrm{q}$ & & & \\
\hline ESA & Energetic electrons & $5 \mathrm{eV}-22 \mathrm{keV} / \mathrm{q}$ & & & \\
\hline EIS & High energy particles & $40-500 \mathrm{keV}(\mathrm{e}, \mathrm{p}, \mathrm{He}, \mathrm{O})$ & & & \\
\hline XUV & EUV spectrometer & $\mathrm{He}^{+}$ion contents & & & \\
\hline PWA & & & 7.7 & Including & \\
\hline PWS & Sounder and HF waves & Electron density profile, HF waves & & 4 antennas & \\
\hline LFA & Plasma waves & VLF/ELF waves & & & \\
\hline MPM & & & 8.8 & Including & \\
\hline MGF & Magnetic field & 3 -axis, $\pm 16 \mathrm{nT}$ to $\pm 65536 \mathrm{nT}$ & & 5-m boom & \\
\hline PET & Electron temperature & Electron temperature probe & & & \\
\hline UVS & UV spectra & $\mathrm{H}, \mathrm{O}, \mathrm{CO}, \mathrm{CO}_{2}$ imaging, $\mathrm{D} / \mathrm{H}$ ratio & & & \\
\hline MIC & Visible camera & Visible images, 3 colors & 3.1 & & France \\
\hline TPA & Thermal ion drift & $0.1-100 \mathrm{eV}$, Drift velocity & 4.0 & & Canada \\
\hline IMI & Energetic ion mass & $0.5-40 \mathrm{keV} / \mathrm{q}$ & 3.0 & & Sweden \\
\hline MDC & Dust counter & Dust counter & 0.7 & & Germany \\
\hline NMS & Neutral gas mass & Neutral gas mass spectrometer & 2.6 & & U.S.A. \\
\hline $\begin{array}{l}\text { USO } \\
\text { (Radio sC }\end{array}$ & $\begin{array}{l}\text { Ultrastable oscillator } \\
\text { ce) }\end{array}$ & Radio science & 0.5 & & U.S.A. \\
\hline
\end{tabular}

\section{Scientific Instruments}

The 15 scientific instruments listed in Table 2 are carried by PLANET-B. Some of instruments shares a common I/F and power unit to reduce component size and mass. For example, the control units of ISA, ESA, EIS, and XUV instruments are installed in one component (PSA-E where PSA stands for Plasma Spectrum Analyzer). Among the 15 scientific instruments, 6 instruments have been designed under the international collaboration.

Some of them will make observations in the parking orbit around Earth (July-December, 1998) and during the cruising phase to Mars (December 1998-October 1999). But the fullscale observations will start after the deployment of antennas and boom that will be done shortly after the insertion of the spacecraft into Mars orbit in October 1999.

\subsection{Upper atmosphere and ionosphere}

The neutral mass spectrometer (NMS) and the ion mass spectrometers (TPA and IMI) measures the composition of neutral atmosphere and ionospheric ions, respectively, along the orbital track of the spacecraft, while the ultra-violet imaging photometer (UVS) observes some of constituents remotely. These instruments provide us with the fundamental constituents of the neutral upper atmosphere and ionosphere of Mars.

One of the argument made for the presence of the Martian magnetic field is the low ionospheric pressure measured by the Viking probe compared with the solar wind dynamic pressure (Luhmann and Brace, 1991). The remote sensing of electron density obtained by the topside sounder technique, as well as in situ measurements (PWS), will give us crucial information on the altitude profile of the Martian ionosphere. Together with electron temperature measurements by the PET instrument, good estimates of the ionospheric plasma pressure will be made.

Solar wind will transfer a part of its momentum to the ionospheric ions at the interaction with the ionosphere. Neutral wind will also interact with the ions. Although PLANET-B has no instrument to detect neutral wind directly, the thermal ion drift velocity measured by the TPA instrument can be used to construct dynamical features of the neutral upper atmosphere and ionosphere. Effective collisions given rise by various plasma waves would play important roles in the solar wind interaction with the ionosphere. Many of particle acceleration/thermalization processes are known to involve waveparticle interactions around the Earth environment. Similar or unknown processes relating to wave-particle interactions would be operating in driving ionospheric ion escapes. Lightning activity associated with dust storms is expected in the lower atmosphere which could emit electromagnetic waves like whistler waves in the Earth. Plasma waves in the ELF to HF range are measured by the PWS and LFA instruments. 


\subsection{Ion pickup and magnetotail}

The solar wind approaching to Mars will first encounter hot upper atmospheric atoms before reaching the main body of the ionosphere. Some of these atoms will be ionized with the charge exchange and/or solar UV in the solar wind region, and be transported with the solar wind. This is called the ion pickup process. Its rate has not been measured at Mars experimentally. The small radius of Mars compared with the gyro-radius of pickup ions should produce asymmetric distribution of energetic ions in the dayside solar wind region and the nightside magnetotail (Luhmann, 1990). The EIS and high energy channel of the IMI and ISA instruments will observe these pickup ions.

An interaction with the solar wind produces energetic electrons and ions of a few hundreds of $\mathrm{eV}$ to a few tens of $\mathrm{keV}$. The energy of these plasma particles depends on the mass of the particles and the solar wind condition including an orientation of magnetic field. The escaping oxygen ion beam found in the magnetotail by Phobos 2 has been accelerated up to a few $\mathrm{keV}$ (Lundin et al., 1990). Measurements of energy spectra, pitch angle distribution and mass will be carried out by the combination of the ESA, ISA, IMI, and EIS instruments.

Recently, Luhmann and Kozyra (1991) pointed out that more than half of pickup oxygen ions reimpact the atmosphere of Mars. Through the reimpact process, atmospheric neutral particles gain an energy and consequently a significant amount of neutral atoms are lost. This is a sputtering loss process. The UVS instrument will have a possibility to detect these atoms.

\subsection{Magnetic field}

If Mars has an intrinsic magnetic field of the order of $50 \mathrm{nT}$ at the ionospheric altitude, the interaction of the solar wind with the ionosphere would become quite different from that at Venus. Presence of the intrinsic magnetic field also suggests that the planetary dynamo action in the Martian core is still operating. This imposes a bounding condition to the thermal history of Mars. Even when the intrinsic magnetic field is absent, the solar wind magnetic field would pile up above the dayside ionosphere and drape toward the nightside of Mars forming a Martian magnetotail. The magnetic field sensor (MGF) is deployed on a boom $5 \mathrm{~m}$ from the spacecraft to avoid interference from the spacecraft main body. Magnetic field measurements at low altitudes, especially in the nightside ionosphere, will give a final resolution of the magnetic moment of Mars.

Remnant magnetic field is an interesting issue. Magnetic field variations along the spacecraft track near periapsis will give us some information on magnetic environment at Mars in the past. Combination of surface images obtained by the MIC instrument with magnetic field variations will make it possible for us to evaluate the intrinsic magnetic field of Mars in the past.

Items above described are intended mainly for the study of Martian aeronomy and the interaction with the solar wind. The following measurements aim at contributing to other research areas.

\subsection{Dust environment}

Dust distribution in the space between the Earth and Mars is measured by the dust counter (MDC) along the trans-Mars orbit. The MDC instrument is also used for the detection of dust rings which might exist along the orbits of the Martian satellites, Phobos and Deimos. It will, at least, give us information on a possible maximum density of dust rings around Mars.

\subsection{Visible images and surface reflectivity at radio fre- quency}

As a monitor for the meteorological activities in the lower atmosphere that could modify the upper atmosphere significantly, a visible camera (MIC) of moderate resolution is installed. This camera will be used to take global images of Mars from a large distance at least once per orbital period. This image will give us crude information of cloud distribution, polar haze activity, dust storms, and growth and decay of polar ice.

The camera is also used for the surface science from taking images near the periapsis altitude with the resolution of $100 \mathrm{~m}$. It has a capability of taking 3-color images by choosing filters, which give us some information on the surface materials. Selecting targets of interest, high-resolution images will be also taken. A study of weathering effects is one of the research targets which can be done by comparing with the Viking images. The orbit of PLANET-B intersects those of Phobos and Deimos and we will have several chances of close encounters with those satellites. It is planned to take close-up images of them at the encounters with an autonomous imaging technique (Kawaguchi et al., 1995).

The RF radio waves transmitted by the PWS instrument penetrate the ionosphere at the high frequency end and surface echo will be received at these frequencies. Analysis of surface echo is expected to give some information on the sub-surface structure and materials of Mars. A good reflectivity may indicate the presence of water in the sub-surface region. With a combination of surface echo measurements and images from the MIC, relationships between sub-surface materials and surface structures will be obtained.

\subsection{Observations of minor constituents}

Interplanetary glow at Helium II line $(30.4 \mathrm{~nm})$ is measured during the cruise phase by the XUV instrument. This measurement is included primarily to provide basic data for future magnetospheric imaging technology, but it also give Helium ion content in the Martian upper atmosphere. Helium content is one of the key parameters for the study of the thermal history of Mars.

Deuterium (D) and hydrogen $(\mathrm{H})$ ratio in the upper atmosphere is one of the key information on the evacuation of water from the Martian atmosphere. $\mathrm{D} / \mathrm{H}$ ratio is measured by the absorption cell technique as a part of the UVS instrument.

Science objectives of the program are briefly described. Figure 1 schematically summarizes observation strategy. Descriptions of each instrument and its key sciences are found in companion papers in this issue.

\section{Orbit Design Strategy}

The launch vehicle for PLANET-B is the 2nd of the M-V series. The $\mathrm{M}-\mathrm{V}$ is a new type of solid-motor rocket, aiming at a larger payload capability required for space sciences in the late 1990 s and the early 21 st century. It has a launch capability of approximately $2000 \mathrm{~kg}$ payload into low-Earth 


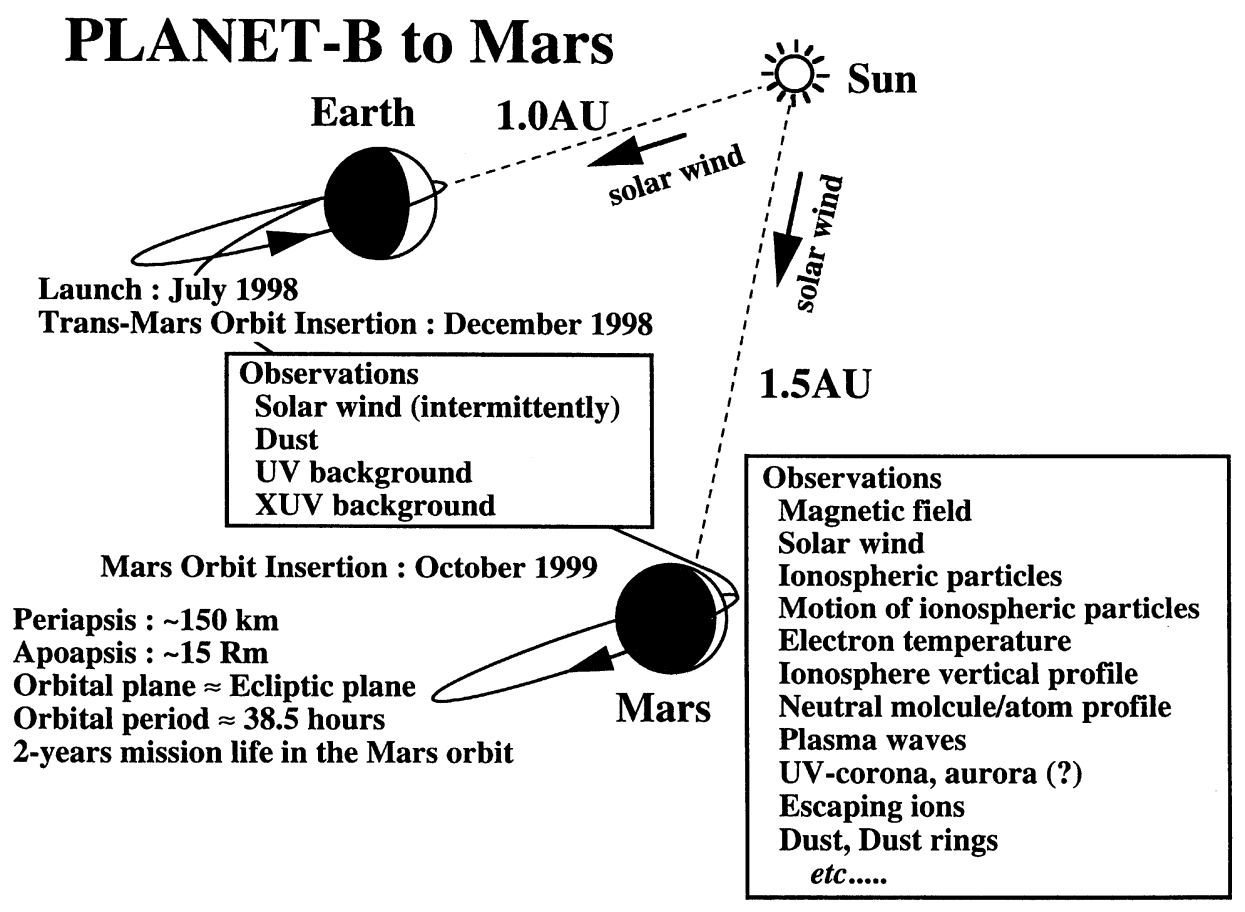

Fig. 1. Observation targets after the launch of PLANET-B.

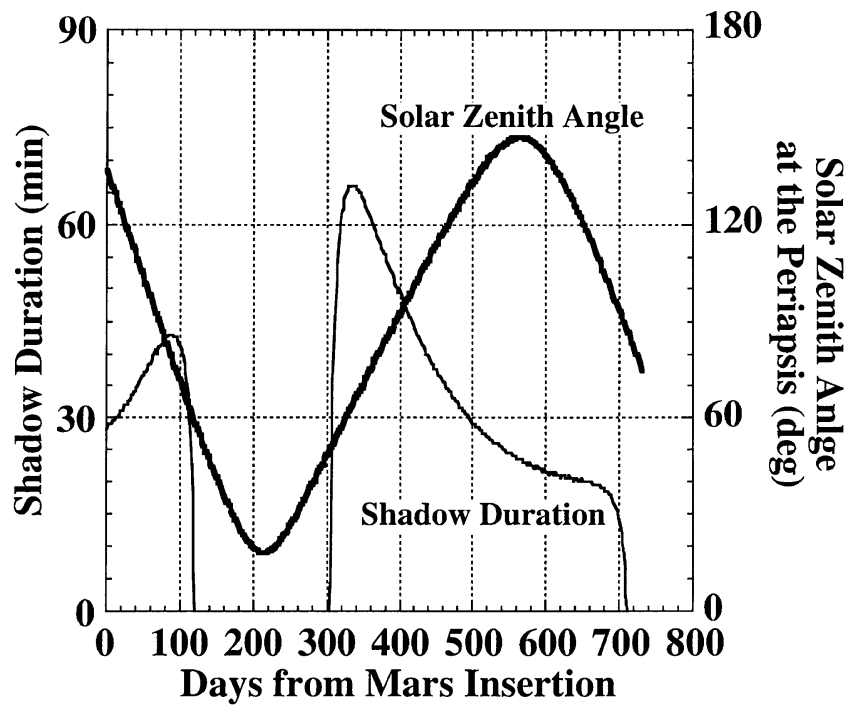

Fig. 2. Variations of Sun-eclipse time interval and solar zenith angle at the periapsis after the Mars orbit insertion.

orbit. The first vehicle was successfully launched in February 1997, putting the MUSES-B (HALCA) spacecraft into an elliptical orbit around the Earth.

After the launch in July 1998, PLANET-B will stay for 4 months in a parking orbit with the apogee of about 400,000 $\mathrm{km}$ and the perigee of $7,000 \mathrm{~km}$. To reduce the propellant consumption at the trans-Mars orbit insertion which will be made in December 1998, a lunar-swing-by technique will be used during the parking orbit. This technique has been established by the missions HITEN and GEOTAIL.
After about a 10 month voyage along the trans-Mars orbit (the cruising phase), the spacecraft will be inserted into a Mars revolving orbit in October 1999, with an initial periapsis altitude of about $300 \mathrm{~km}$ and an apoapsis of 15 Mars radii. The inclination will be about 170 degrees with respect to the ecliptic plane. This orbit has been designed to meet both the science requirements and the engineering restrictions. One of the most important engineering requirements is to keep the sun-shadow interval minimum to reduce the battery weight. The key requirement from the science objectives is to make the solar zenith angle at the low altitudes as small as possible. This requirement came up to realize observations near the sub-solar ionosphere. After the determination of the orbit parameters, periapsis altitudes will be decreased as low as $150 \mathrm{~km}$. Figure 2 shows the planned sun-shadow duration and the solar zenith angle at periapsis during the mission life, where the flight time indicates an elapsed time from Mars orbit insertion. The maximum sun-shadow time period for this scenario is shorter than 70 minutes.

\section{Operation Strategy for Science Observations in Mars Orbit}

Since the spacecraft speed is the highest during the low altitude measurements around the periapsis, a higher edition bit rate is requested to obtain detailed structures of the upper atmosphere and ionosphere. At higher altitudes, continuous observations are necessary to figure out the magnetotail, bow shock and upstream region. They are the basic requirements from the scientific instruments to maximize science outputs. The telemetry rate of X-band is $2 \mathrm{kbps}$ or $4 \mathrm{kbps}$ for most of time during the mission life. It depends on the Earth-Mars distance. This gives some limitations on science observations. To overcome the limitations, most of scientific 
Table 3. PLANET-B SWG core members.

\begin{tabular}{lll}
\hline Researcher & Organization & Title or PI \\
\hline K. Tsuruda & Inst. Space and Astron. Sci. & Program Manager \\
I. Nakatani & Inst. Space and Astron. Sci. & Project Manager \\
T. Yamamoto & Inst. Space and Astron. Sci. & Project Scientist, PI of MGF \\
H. Hayakawa & Inst. Space and Astron. Sci. & PI of ISA \\
S. Machida & Kyoto Univ. & PI of ESA \\
J. Kikuchi & Waseda Univ. & PI of EIS \\
R. Lundin & Swedish Inst. Space Phys. & PI of IMI \\
A. W. Yau & Univ. Calgary & PI of TPA \\
H. B. Niemann & NASA/GSFC & PI of NMS \\
K. Oyama & Inst. Space and Astron. Sci. & PI of PET and Radio Science \\
T. Ono & Tohoku Univ. & PI of PWS \\
H. Matsumoto & Kyoto Univ. & PI of LFA \\
H. Fukunishi & Tohoku Univ. & PI of UVS \\
K. Yamashita & Nagoya Univ. & PI of XUV \\
T. Mukai & Kobe Univ. & PI of MIC \\
E. Igenberg & Tech. Univ. Munich & PI of MDC \\
S. Sasaki & Univ. Tokyo & PI of MDC \\
H. Shinagawa & STE lab., Nagoya Univ. & PI of Theory and Modeling \\
\hline
\end{tabular}

instruments use onboard data compression. The onboard data recorder is utilized to realize maximum science output. In principle, $65 \mathrm{kbps}$ or $32 \mathrm{kbps}$ data rate will be used to record data obtained at low altitudes, below $1000 \mathrm{~km}$. In higher altitudes, the data recorder will record at $512 \mathrm{bps}$ or 256 bps. Recorded data are reproduced when the ground station links the spacecraft. In this way, continuous data will be obtained with high time resolution at lower altitudes.

\section{Science Data Management and Archiving}

The planning for observations and the policy of data usage are discussed and decided at the PLANET-B science working group meetings (PLANET-B SWG meeting) which consists of the principal investigators, co-investigators, and those responsible for the PLANET-B program. The PLANET-B SWG meeting is chaired by the project scientist.

The raw data acquired from the spacecraft are archived in the project data system at the Institute of Space and Astronautical Science (ISAS) and distributed to the experimenter groups along with ancillary data. The evaluation of raw data is the responsibility of the respective principal investigator and his group. The evaluated data are to be returned and registered in the ISAS data system. All data obtained by the PLANET-B project are considered as common assets not only for the PLANET-B scientists, but also for anyone interested in the data. Time and method of data release for common use are decided by the PLANET-B SWG meeting. Those who are interested in the data obtained in the early phase (data evaluation phase), are strongly encouraged to join in the SWG by contacting with an appropriate principal investigator or the project scientist. Table 3 shows core members of the PLANET-B SWG.

\section{Summary}

The scientific objectives and outlines of the program are briefly described. The test of the prototype models were finished in 1995. The results of the engineering challenges have become clear during the test. The flight model design started in 1995. The first electrical/mechanical test of the flight model units finished in June 1997, without any fatal problems. The final flight model test will begin in October 1997, and the spacecraft is scheduled to launch in July 1998 from Kagoshima Space Center. During the Earth parking orbit phase, the lunar-swing-by technique is introduced to reduce a propellant consumption at the insertion of transMars orbit, which will be made in December 1998. The Mars orbit insertion will be carried out in October 1999, and from then full-observations aiming at the understandings of the Martian upper atmospheric environment will start.

Acknowledgments. The authors thanks to all members of PLANET-B to realize low-mass and high-performance instruments. Special thanks are expressed to I. Nakatani for his efforts in managing the project.

\section{References}

Hanson, W. B., S. Sanatani, and D. R. Zuccaro, The Martian ionosphere as observed by the Viking retarding potential analyzers, J. Geophys. Res., 82, 4,351-4,363, 1977. 
Kawaguchi, J., T. Hashimoto, I. Nakatani, and K. Ninomiya, Autonomous imaging of Phobos and Deimos for the PLANET-B mission, J. Guidance, Control, and Dynamics, 18, 605-610, 1995.

Luhmann, J. G., A model of the ion wake of Mars, Geophys. Res. Lett., 17, 869-872, 1990.

Luhmann, J. G. and L. H. Brace, Near-Mars space, Rev. Geophys., 22, 121 $140,1991$.

Luhmann, J. G. and J. U. Kozyra, Dayside pickup oxygen ion precipitation at Venus and Mars: spatial distributions, energy deposition, and consequences, J. Geophys. Res., 96, 5457-5467, 1991.

Lundin, R., A. Zakharov, R. Pellinen, H. Borg, B. Hultqvist, N. Pissarenko, E. M. Dubinin, S. W. Barabash, I. Liede, and H. Koskinen, First measurements of the ionospheric plasma escape from Mars, Nature, 341, 609-612, 1989.

Lundin, R., A. Zakharov, R. Pellinen, S. W. Barabasj, H. Borg, E. M. Dubinin, B. Hultqvist, H. Koskinen, I. Liede, and N. Pissarenko, ASPERA/PHOBOS measurements of the ion outflow from the Martian ionosphere, Geophys. Res. Lett., 17, 873-876, 1990.

Slavin, J. A. and R. E. Holzer, Solar wind flow about the terrestrial planets 1.
Modeling bow shock position and shape, J. Geophys. Res., 86, 11,401$11,418,1981$

Slavin, J. A., R. E. Holzer, J. R. Spreiter, S. S. Stahara, and D. S. Chaussee, Solar wind flow about the terrestrial planets 2. Comparison with gas dynamic theory and implications for solar-planetary interactions, J. Geophys. Res., 88, 19-35, 1983.

Taylor, H. A., Jr., H. C. Brinton, S. Bauer, R. E. Hartle, P. A. Cloutier, F. C. Michel, R. E. Daniell, Jr., T. M. Donahue, and R. C. Maehl, Ionosphere of Venus: first observations of the effects of dynamics on the dayside ion composition, Science, 293, 755-757, 1979.

Tsuruda, K., I. Nakatani, and T. Yamamoto, PLANET-B mission to Mars 1998, Adv. Space Res., 17, No. 12, 21-29, 1996.

Zhang, T.-L., J. G. Luhmann, and C. T. Russell, The solar cycle dependence of the location and shape of the Venus bow shock, J. Geophys. Res., 95, 14,961-14,967, 1990.

T. Yamamoto and K. Tsuruda (e-mail: tsuruda@stp.isas.ac.jp) 